ARTICLE

https://doi.org/10.1038/s41467-019-08613-3

\title{
OPEN
}

\section{Revealing the role of lattice distortions in the hydrogen-induced metal-insulator transition of $\mathrm{SmNiO}_{3}$}

\author{
Jikun Chen ${ }^{1}$, Wei Mao ${ }^{2}$, Binghui Ge (10 ${ }^{3}$, Jiaou Wang ${ }^{4}$, Xinyou Ke ${ }^{5}$, Vei Wang ${ }^{6}$, Yiping Wang ${ }^{7}$, Max Döbeli ${ }^{8}$, \\ Wentong Geng (1) ${ }^{1}$, Hiroyuki Matsuzaki ${ }^{2}$, Jian Shi ${ }^{7}{ }^{7} \&$ Yong Jiang ${ }^{1}$
}

The discovery of hydrogen-induced electronic phase transitions in strongly correlated materials such as rare-earth nickelates has opened up a new paradigm in regulating materials' properties for both fundamental study and technological applications. However, the microscopic understanding of how protons and electrons behave in the phase transition is lacking, mainly due to the difficulty in the characterization of the hydrogen doping level. Here, we demonstrate the quantification and trajectory of hydrogen in strain-regulated $\mathrm{SmNiO}_{3}$ by using nuclear reaction analysis. Introducing $2.4 \%$ of elastic strain in $\mathrm{SmNiO}_{3}$ reduces the incorporated hydrogen concentration from $\sim 10^{21} \mathrm{~cm}^{-3}$ to $\sim 10^{20} \mathrm{~cm}^{-3}$. Unexpectedly, despite a lower hydrogen concentration, a more significant modification in resistivity is observed for tensile-strained $\mathrm{SmNiO}_{3}$, substantially different from the previous understanding. We argue that this transition is explained by an intermediate metastable state occurring in the transient diffusion process of hydrogen, despite the absence of hydrogen at the post-transition stage.

\footnotetext{
${ }^{1}$ Beijing Advanced Innovation Center for Materials Genome Engineering, School of Materials Science and Engineering, University of Science and Technology Beijing, 100083 Beijing, China. ${ }^{2}$ School of Engineering, the University of Tokyo, 2-11-16 Yayoi, Bunkyo-ku, Tokyo 113-0032, Japan. ${ }^{3}$ Beijing National Laboratory for Condensed Matter Physics, Chinese Academy of Sciences, 100190 Beijing, China. ${ }^{4}$ Beijing Synchrotron Radiation Facility, Institute of High Energy Physics, Chinese Academy of Sciences, 100049 Beijing, China. ${ }^{5}$ Department of Mechanical and Aerospace Engineering, Case Western Reserve University, Cleveland, OH 44106, USA. ${ }^{6}$ Department of Applied Physics, Xi'an University of Technology, 710054 Xi'an, China. ${ }^{7}$ Department of Materials Science and Engineering, Rensselaer Polytechnic Institute, Troy, New York, NY 12180, USA. ${ }^{8}$ Laboratory of lon Beam Physics, ETH Zurich, CH-8093 Zurich, Switzerland. These authors contributed equally: Jikun Chen, Wei Mao. Correspondence and requests for materials should be addressed to

J.C. (email: jikunchen@ustb.edu.cn) or to J.S. (email: shij4@rpi.edu) or to Y.J. (email: yjiang@ustb.edu.cn)
} 
ecently, ion-induced quantum phase transitions have been demonstrated in several strongly correlated materials systems that have interesting applications in enabling artificial intelligence ${ }^{1-3}$, high-performance energy conversion ${ }^{4}$, biological sensing ${ }^{5}$, and multifunctional logic/memory devices ${ }^{6}$. In contrast to conventional semiconductors, $d$-orbital-band-correlated systems usually exhibit extremely complex electronic configurations and phase diagrams, which can be varied abruptly upon either electrostatic $^{5,6}$ or chemical doping ${ }^{1,2}$. Recent discoveries were demonstrated in correlated nickelates ${ }^{1,2,4,5}, \mathrm{SrCoO}_{\mathrm{x}}{ }^{6}$ and $\mathrm{VO}_{2}{ }^{7}$, in which either strong correlations, such as the Mott-Hubbard U, could be manipulated to enable metal-insulator transition (MIT) or electronic magnetic structures could be tuned to reach multiple magnetic ground states.

Among these ion species, the proton/hydrogen system is a particularly intriguing one due to its fast kinetics, ubiquitous presence, and mid-value electronegativity. Proton/hydrogen interactions with $3 d$-band-electron-correlated oxide materials and the resultant phase transitions, e.g., Mott-Hubbard among multiple electronic phases has brought up an exciting direction for exploring new physics and emerging multifunctional electronic devices $^{1-7}$. However, despite these technical breakthroughs, a critical question remains unsolved: what is the fundamental microscopic mechanism leading to the correlated phase transition? Owing to their small atomic weight and high volatility, the presence of a hydrogen atom $\left({ }^{1} \mathrm{H}\right)$ or proton $\left({ }^{1} \mathrm{H}^{+}\right)$is rather difficult to directly quantify. In one previous investigation, the isotope of the deuterium $\left({ }^{2} \mathrm{D}\right)$ isotope rather than ${ }^{1} \mathrm{H}$ is used to demonstrate the physical presence of hydrogen within electrochemically hydrogenated $\mathrm{SrCoO}_{3}{ }^{6}$. Nevertheless, despite the similarity in chemical properties for ${ }^{1} \mathrm{H}$ and ${ }^{2} \mathrm{D}$, their physical properties, such as density, diffusion kinetics, and volatility, are considerably different $t^{8,9}$. For other $d$-band-correlated systems, such as hydrogenated $\mathrm{SmNiO}_{3}$, although a sharp increase in the resistivity by eight orders of magnitude was observed upon proton doping ${ }^{1,2}$, a solid experiment-verified atomic understanding of the role of protons has not yet been presented.

In previous investigations, the hydrogen-induced highly insulating state of $\mathrm{SmNiO}_{3}$ was hypothetically attributed to the transformation from the electron itinerant orbital state $N i^{3+} t_{2 g}^{6} e_{g}^{1}$ to the electron-localized state $N i^{2+} t_{2 g}^{6} e_{g}^{2}$ owing to the hydrogen doping $1,2,4,5$. Nevertheless, this understanding omitted the role of possible subtle structural phase transition, which appear to be inevitable but beyond the resolution of most instruments. For example, it may be possible that the orbital reconfiguration of nickel is also achievable via kinetic processes during hydrogenation even without the physical presence of (or doping by) the hydrogen elements. This is seen previously from the irreversibility of hydrogen-induced defect behaviors, such as hydrogen embrittlement $^{10,11}$, cavitation/blistering ${ }^{12}$, and interface failure ${ }^{13}$, owing to the defects that are formed or developed in the hydrogen diffusion path ${ }^{10-15}$. Finding the root causes of hydrogen-induced switching in correlated electron states by quantifying the hydrogen composition is critical within the field of hydrogen-induced strong correlations in condensed matter.

In this article, we demonstrate that the electron-localized insulating state observed in the hydrogenated $\mathrm{SmNiO}_{3}$ under tensile strain is more likely a direct consequence of a proton incorporation/removal-induced transient chemical state (a proton-induced oxygen-removed metastable state), via direct ${ }^{1} \mathrm{H}$ quantification by using nuclear reaction analysis (NRA). Introducing tensile lattice distortions to single crystalline $\mathrm{SmNiO}_{3}$ films is shown to reduce the hydrogen incorporation concentration but elevates the hydrogen-induced electron localization and the resistivity. Near edge X-ray absorption fine structure (EXAFS) and Rutherford backscattering (RBS) analysis indicate a more distinct transition in the orbital configuration from an electron itinerant to electron-localized state is achieved in tensile-distorted $\mathrm{SmNiO}_{3}$ that is not related to the final hydrogen composition. The transition is instead associated with the transient chemical state formed in the transient diffusion process of hydrogen, despite the absence of hydrogen at the post-transition stage.

\section{Results}

Quantifying hydrogen via nuclear reaction analysis. To date, the only way to directly quantify the absolute amount of ${ }^{1} \mathrm{H}$ isotope within a solid is to use NRA, based on the nuclear resonance reactions between high-energy $(\mathrm{MeV}){ }^{15} \mathrm{~N}^{2+}$ ions from an accelerator with ${ }^{1} \mathrm{H}$-releasing detectable gamma-rays ${ }^{16-18}$. As illustrated in Fig. 1a, to probe the hydrogen concentration located at various depth, the hydrogenated $\mathrm{SmNiO}_{3}$ was irradiated by ${ }^{15} \mathrm{~N}^{2+}$ incident ion beams at different kinetic energies $\left(E_{\mathrm{K}}\right)$. The nuclear resonance with hydrogen can only take place when the $E_{\mathrm{K}}$ of the ${ }^{15} \mathrm{~N}^{2+}$ after traversing in solid ( $E_{\mathrm{K}}$ loss of $3.1945 \mathrm{keV} \mathrm{nm}^{-1}$ for $\mathrm{SmNiO}_{3}$ ) is within a narrow range of $\sim 6.385 \mathrm{MeV}^{18}$. By varying the $E_{\mathrm{K}}$ of the incident ${ }^{15} \mathrm{~N}^{2+}$ ion beam from $6.3 \mathrm{MeV}$ to $7.3 \mathrm{MeV}$ and measuring the respective yield of gamma-ray intensity from the resonance ${ }^{16}$, the concentration of hydrogen as a function of the allocation depth within $\mathrm{SmNiO}_{3}$ is obtained. More details of the quantification of the hydrogen concentration from the NRA spectrum are given in the Supplementary Methods.

Strain regulation for $\mathrm{SmNiO}_{3}$. Figure 2a illustrates the general concept for regulating the stabilized hydrogen incorporation concentration within the hydrogenated $\mathrm{SmNiO}_{3}$. More details on the electrode configuration and pattern can be found on Supplementary Figure 1 . When exposing the hydrogenated $\mathrm{SmNiO}_{3}$ in the air, the dehydrogenation potential to drag out the hydrogen (or proton) from $\mathrm{H}_{\mathrm{x}} \mathrm{SmNiO}_{3}$ via the platinum catalyst counteracts with the crystal trapping potential (the thermal dynamical and dynamical barrier for hydrogen to diffuse out of the oxide) to impede the dehydrogenation, as illustrated in Fig. 2a. The crystal trapping potential is mainly associated to the energy barrier $\left(E_{\mathrm{A}}\right)$ for the incorporated hydrogen to overcome before being dragged out of the crystal by the oxygen molecule in the air. The $E_{\mathrm{A}}$ is expected to be reduced when imposing a biaxial tensile strain upon $\mathrm{SmNiO}_{3}$, owing to the reduced repulsion between the $\mathrm{Ni}$ site with the proton to improve the rotational diffusion of proton and further transfer to neighboring oxygen, as illustrated in Fig. 2b. To further demonstrate how tensile distortion affects the migration of hydrogen in $\mathrm{SmNiO}_{3}$, the energy migration barrier under different tension strain by using density functional theory (DFT)-based Vienna Ab initio Simulation Package was calculated. As more details in Supplementary Discussions, imparting a tensile distortion to $\sim 2 \%$ reduces the diffusion energy barrier and increases the transition rate of hydrogen by 14 times at room temperature. Our calculation is in well agreement with the previous reports ${ }^{19-21}$ that an enhanced proton diffusion coefficient as previously observed in tensile-strained proton conducting oxides. Owing to the reduced $E_{\mathrm{A}}$ as illustrated in Fig. $2 \mathrm{c}$ for dehydrogenation, a smaller hydrogen composition is expected for more tensile-strained $\mathrm{SmNiO}_{3}$ via the same processes of hydrogenation followed by an exposure in air.

To introduce as-proposed biaxial lattice distortion, the single crystalline $\mathrm{SmNiO}_{3}$ thin films were co-lattice grown on $\mathrm{SrTiO}_{3}$ (001), ( $\mathrm{La}, \mathrm{Sr})(\mathrm{Al}, \mathrm{Ta}) \mathrm{O}_{3}$ (001), or $\mathrm{LaAlO}_{3}$ (001) substrates by pulsed laser deposition. The quasi-single crystal structure of asgrown $\mathrm{SmNiO}_{3}$ with the same orientation to the substrate is 
a

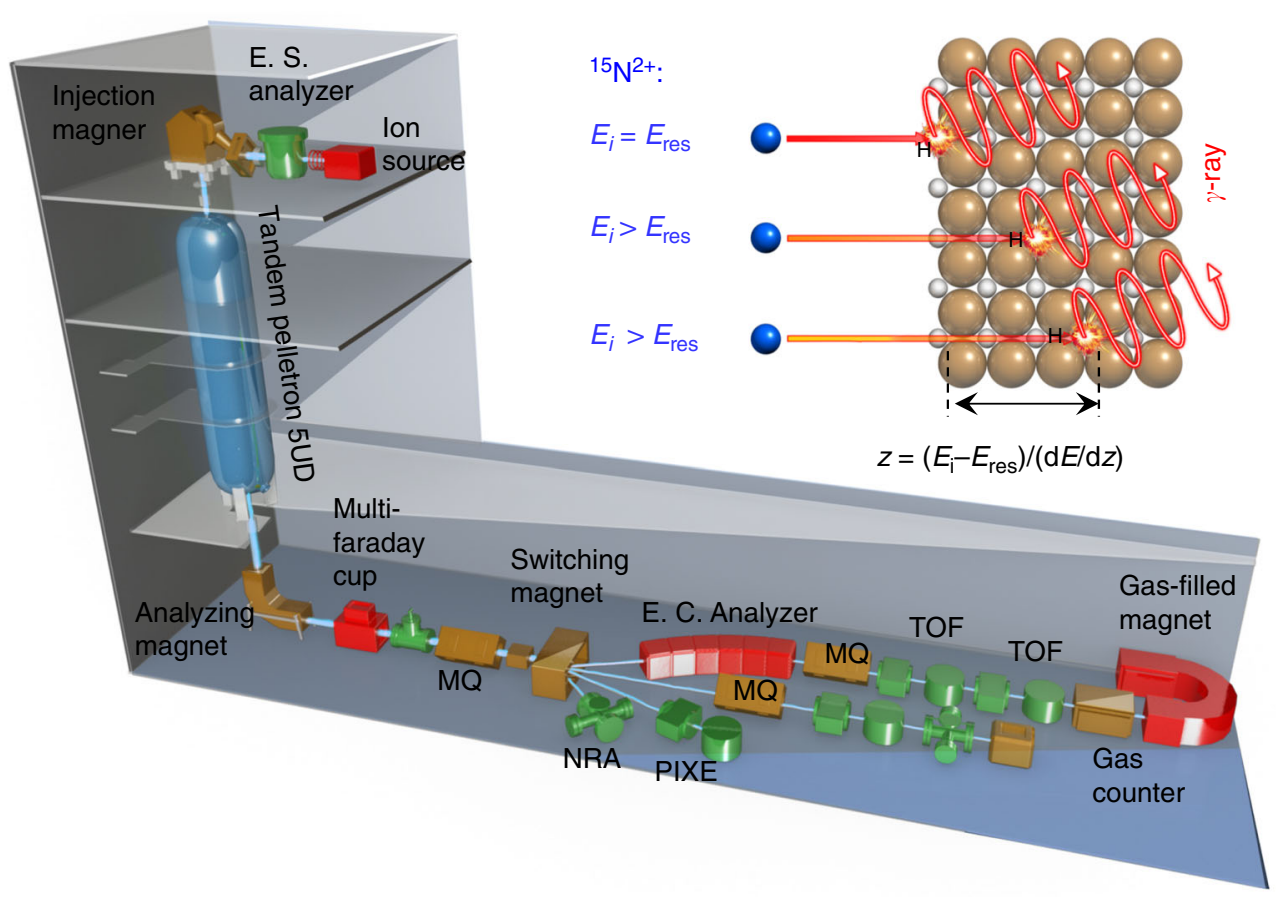

Fig. 1 Illustration of the nuclear reaction analysis. a Schematic illustration of the accelerator system for the nuclear reaction analysis (NRA). The system consists of components, such as the ion source, electrostatic spherical (E.S.) analyzer, injection magnet, electrostatic cylindrical (E.C.) analyzer, several magnetic quadrupole-lens (M.Q.), time of flight (TOF), and analyzing cambers. In the present work, the system is mainly used for the NRA measurement, while the ultra high vacuum NRA, Rutherford backscattering (RBS), elastic recoil detection analysis (ERDA), and particle induced X-ray emission (PIXE) can be also performed in additional chambers and altering the ion source. $\mathbf{b}$ The working principle for quantitative detection of the hydrogen composition as a function of distribution depth by using nuclear reaction analysis (NRA). Only ${ }^{15} \mathrm{~N}^{2+}$ ions with kinetic energies $\left(E_{\mathrm{K}}\right)$ of $6.385 \mathrm{MeV}$ after penetrating SmNiO${ }_{3}$ (Energy loss: $3.1945 \mathrm{keV} \mathrm{nm}^{-1}$ ) can be resonant with the hydrogen element and yield detectable gamma-rays

demonstrated by their X-ray diffraction (XRD) pattern as shown in Supplementary Figure 2a-f. The difference in lattice constant between the substrate and co-lattice-grown thin films imparts interfacial stains and distorts the lattice of $\mathrm{SmNiO}_{3}{ }^{22-26}$. The strained perovskite phase was stabilized by co-lattice deposition on the template of single crystal substrate lattice ${ }^{22-24}$ that reduces the formation free energy ${ }^{27-29}$.

As shown in Fig. 2d, e, the $\mathrm{SmNiO}_{3}$ exhibits a tensile distortion of $\sim-2.4 \%$ when co-lattice grown on $\mathrm{SrTiO}_{3}$ (001), noticing a smaller lattice constant $\left(a_{0}\right)$ of the film $\left(a_{0, \text { film }}=3.807 \AA\right)$ compared to the substrate $\left(a_{0 \text {,substrate }}=3.905 \AA\right)$. This is confirmed by the reciprocal space mapping (RSM) as shown in Fig. 2d, where the in-plane lattice of $\mathrm{SmNiO}_{3}$ is locked by $\mathrm{SrTiO}_{3}$. Figure $2 \mathrm{e}$ further shows the strain distribution map along both inplane and cross-plane directions, converted from its $\mathrm{RSM}^{30}$. It clearly presents the biaxial tensile distortion of the film material is demonstrated by the expansion of the in-plane lattice by $\sim 2.4 \%$, and the respective cross-plane transverse contraction by $\sim-0.3 \%$. The preservation of tensile distortion at a relatively large magnitude of lattice mismatches may associate to the high tolerance in distorting the $\mathrm{NiO}_{6}$ octahedra as known for the thermodynamically instable rare-earth nickelates. In addition, the variation in kinetic processes when using different deposition approaches is also expected to influence the preservation of the tensile interfacial strain, which was preserved for using $\operatorname{PLD}^{25}$ and relaxed for using chemical vapor deposition ${ }^{31}$. Figure $2 \mathrm{f}$ shows the cross-section morphology of $\mathrm{SmNiO}_{3} / \mathrm{SrTiO}_{3}$ (001) interface, where a co-latticed interface between the single crystalline $\mathrm{SmNiO}_{3}$ film and the $\mathrm{SrTiO}_{3}$ substrate was observed.
As compared to growing on $\mathrm{SrTiO}_{3}$, a reduced tensile distortion of $\sim-1.6 \%$ is expected when growing $\mathrm{SmNiO}_{3}$ on $(\mathrm{La}, \mathrm{Sr})(\mathrm{Al}, \mathrm{Ta})$ $\mathrm{O}_{3}$ (001) substrate $\left(a_{0}=3.87 \AA\right)$, while the $\mathrm{SmNiO}_{3}$ grown on $\mathrm{LaAlO}_{3}(001)$ substrates $\left(a_{0}=3.79 \AA\right)$ is slightly compressive strained up to $\sim 0.4 \%$. Supplementary Figure $2 \mathrm{~g}$ and $\mathrm{h}$ provides the XRD and RSM results for as-grown $\mathrm{SmNiO}_{3} /(\mathrm{La}, \mathrm{Sr})(\mathrm{Al}, \mathrm{Ta}) \mathrm{O}_{3}$ (001) and $\mathrm{SmNiO}_{3} / \mathrm{LaAlO}_{3}(001)$. The surface morphology of asgrown $\mathrm{SmNiO}_{3}$ films on the three difference substrates are demonstrated in Supplementary Figure 3, which shows a reduced surface roughness with an increasing tensile distortion.

Relating $\mathrm{H}$ composition to resistivity for distorted $\mathrm{H}_{x} \mathrm{SmNiO}_{3}$. The same hydrogenation process was performed for the platinum patterned $\mathrm{SmNiO}_{3}$ grown on different substrates similar to the previous reports. In brief, the array of dot-shaped platinum electrodes were deposited on the surface of the $\mathrm{SmNiO}_{3}$ thin films, and the samples were annealed in $1 \% \mathrm{H}_{2} / \mathrm{He}$ gas at $300^{\circ} \mathrm{C}$ for 15-60 min, followed by the same exposure time in the air before NRA (see more details in Supplementary Methods and Supplementary Figure 1). Figure 3a, b shows the hydrogen concentration depth profile before and after the hydrogenation measured by NRA for $\mathrm{SmNiO}_{3} / \mathrm{SrTiO}_{3}$ (001) and $\mathrm{SmNiO}_{3} /$ $\mathrm{LaAlO}_{3}(001)$, respectively, while the ones for $\mathrm{SmNiO}_{3} /(\mathrm{La}, \mathrm{Sr})(\mathrm{Al}$, $\mathrm{Ta} \mathrm{O}_{3}$ (001) is shown in Supplementary Figure 4a. It is worth noticing that intensity of the surface peak in the NRA spectrum is associated to the native hydrogen (i.e., $\mathrm{H}_{2} \mathrm{O}$ ) absorbed by the surface as well as the near surface instrumental function (see more details in Supplementary Figure 4), while the intensity 
a

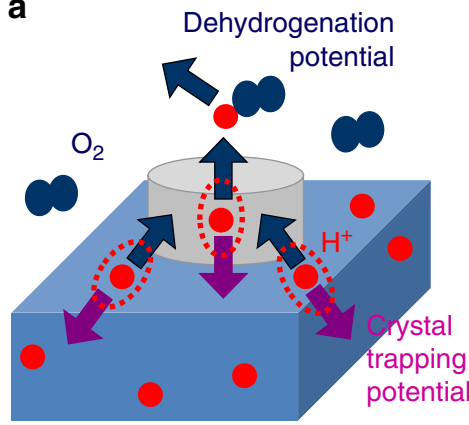

b

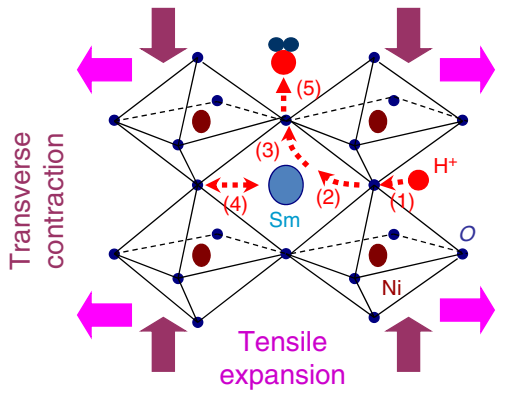

C

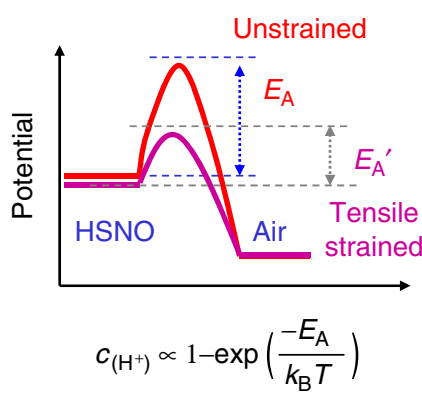

f

f

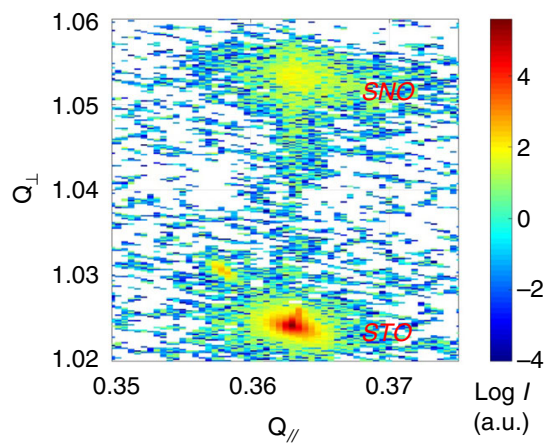

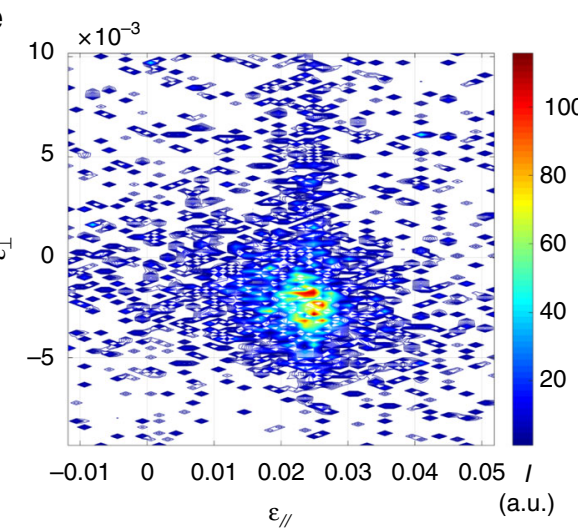

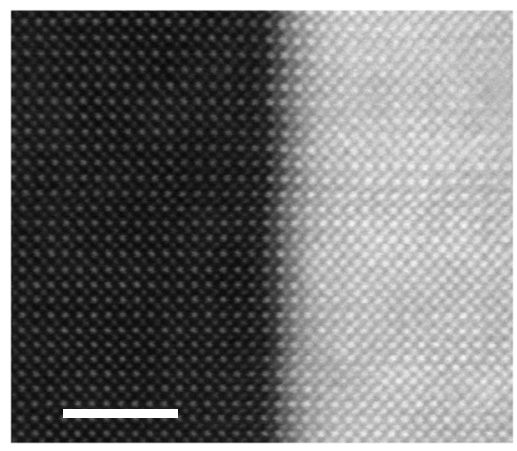

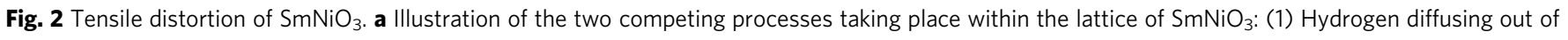
the material driven by the dehydrogenation potential when exposed in an oxygen containing atmosphere, (2) being trapped within $\mathrm{SmNiO}_{3}$ due to the energy barrier provided by the lattice. b Illustrating the proton transport processes within $\mathrm{SmNiO}_{3}$ : (1) proton incorporation, (2) rotational diffusion and (3) transport of protons, (4) bending and stretching of the $\mathrm{Ni}-\mathrm{O}$ bond in the opposite side. Imposing tensile distortion upon $\mathrm{SmNiO}_{3}$ improves step (2) and (3) owing to the elongated $\mathrm{Ni}-\mathrm{O}$, and thereby enhances the proton diffusion coefficient. c Fast diffusion of hydrogen is expected owing to the reduced energy

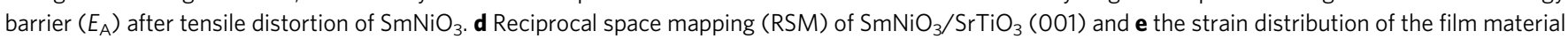
converted from RSM. f Cs-corrected STEM high-angle annular dark-field (HAADF) image of the interface for the $\mathrm{SmNiO}_{3} / \mathrm{SrTiO}_{3}(\mathrm{OO} 1)$. Scale bar is 3 nm

in the bulk material away from the surface is proportional to the hydrogen composition ${ }^{16}$. The hydrogen concentration incorporated within the $\mathrm{SmNiO}_{3}$ film material after annealing for $15 \mathrm{~min}$ saturated at a concentration of $2.3 \times 10^{20} \mathrm{~cm}^{-3}, 3.6 \times 10^{20} \mathrm{~cm}^{-3}$, and $1.4 \times 10^{21} \mathrm{~cm}^{-3}$ when growing on $\mathrm{SrTiO}_{3},(\mathrm{La}, \mathrm{Sr})(\mathrm{Al}, \mathrm{Ta}) \mathrm{O}_{3}$, and $\mathrm{LaAlO}_{3}$, respectively. To exclude the potential proton incorporation or conduction through the $\mathrm{SrTiO}_{3}$ substrate, Supplementary Figure $4 \mathrm{~b}$ shows the NRA spectrum for the hydrogenated $\mathrm{SmNiO}_{3} / \mathrm{SrTiO}_{3}$ sample measured at a larger depth until the interfacial region, and no effective hydrogen signal was observed in the $\mathrm{SrTiO}_{3}$. A reducing tendency in the incorporated hydrogen concentration when enhancing tensile lattice distortion of $\mathrm{SmNiO}_{3}$ was observed, as more clearly demonstrated in Fig. 3c. This is in agreement to our expectation as demonstrated previously. Nevertheless, despite incorporating a smaller hydrogen composition, a more tensile-strained $\mathrm{SmNiO}_{3}$ shows a more significant enhancement in the resistivity after the same hydrogenation process, as shown in Fig. $3 \mathrm{~d}$ (the absolute resistances are shown in Supplementary Figure 5).

The above observation is in contradiction to the previous hypothesis that the metal to insulator transition was triggered by the presence of the hydrogen element within the material lattice ${ }^{1,2,4,5}$. As further demonstrated in Supplementary Figure 6, the resistivity of the hydrogenated $\mathrm{SmNiO}_{3}$ can be fully recovered to the initial magnitude via annealing in $\mathrm{O}_{2}$ at $300^{\circ} \mathrm{C}$ for $30 \mathrm{~min}$, known as a dehydrogenation procedure. Even at room temperature, the resistivity of the hydrogenated $\mathrm{SmNiO}_{3}$ gradually reduced by a long-time exposure in the air. However, by annealing it in the vacuum (pressure below $10^{-3} \mathrm{~Pa}$ ) at $300^{\circ} \mathrm{C}$ for $30 \mathrm{~min}$, the resistivity of the hydrogenated $\mathrm{SmNiO}_{3}$ is further enhanced for both $\mathrm{SmNiO}_{3} / \mathrm{SrTiO}_{3}$ (001) as compared to $\mathrm{SmNiO}_{3} / \mathrm{LaAlO}_{3}$. Annealing in the vacuum will not increase the hydrogen incorporation concentration in $\mathrm{SmNiO}_{3}$, and this is further demonstrated by the NRA measurement where rather low hydrogen concentration was observed for the hydrogenated $\mathrm{SmNiO}_{3}$ sample further annealed in the vacuum.

Transient state. Instead of the physical hydrogen doping effect, the hydrogenation process may also result in a transient chemical state occurring in the transient diffusion process of hydrogen, despite the absence of hydrogen at the post-transition stage. This is further supported by the reduced crystallinity of $\mathrm{SmNiO}_{3}$, as demonstrated by XRD and RSM patterns in this work (see Supplementary Figure 7 and 8), and also observed previously in ref. ${ }^{27}$. It is also worth noticing that a more significant variation in RSM pattern was observed for $\mathrm{SmNiO}_{3} / \mathrm{SrTiO}_{3}$ compared to $\mathrm{SmNiO}_{3} / \mathrm{LaAlO}_{3}$, as shown in Supplementary Figure 8. As for resistivity-temperature relations in both $\mathrm{SmNiO}_{3} / \mathrm{SrTiO}_{3}$ and $\mathrm{SmNiO}_{3} / \mathrm{LaAlO}_{3}$ systems, they can be found on Supplementary Figure 9. It indicates the stretches and distortions of the lattice atoms and bonds are due to the transient diffusion process of hydrogen, and such changes cannot be recovered even by further lowering the hydrogen composition, as illustrated by Fig. 4a. This is kinetically favored since the enhanced disorder of lattice atoms elevates the system entropy and may induce a dip in the free energy of $\mathrm{SmNiO}_{3}(\Delta \mathrm{G}>0)^{27,28}$. By annealing the hydrogenated 
a

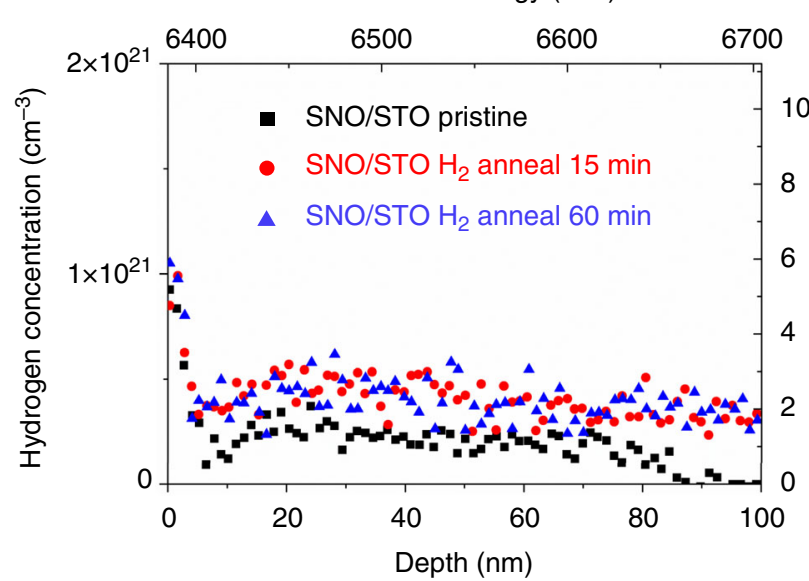

b

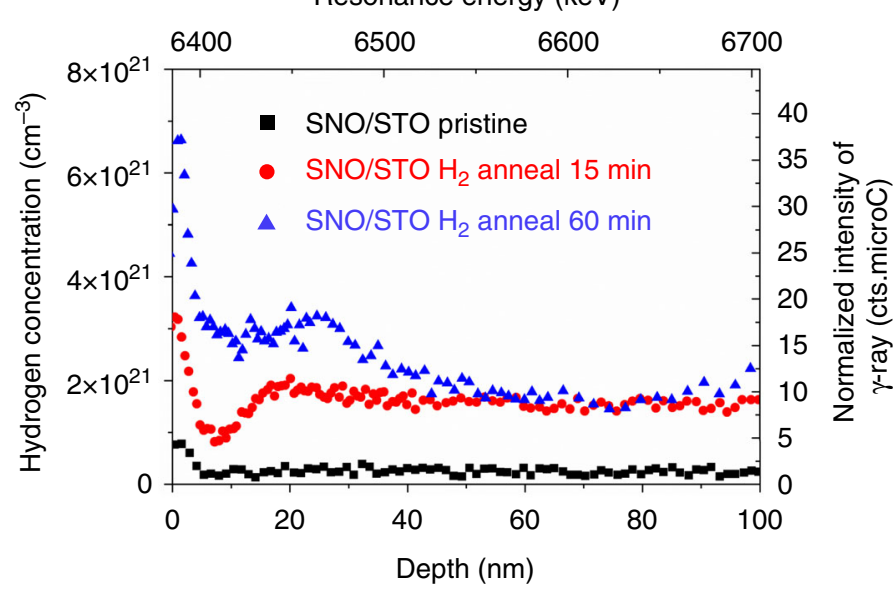

C

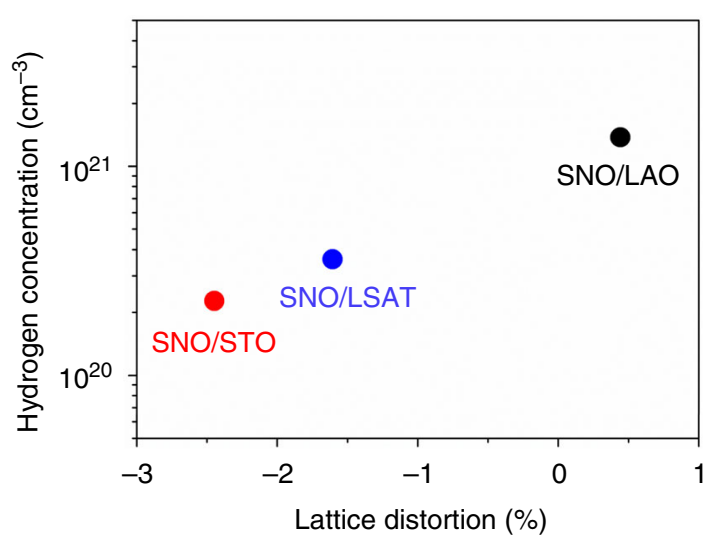

d

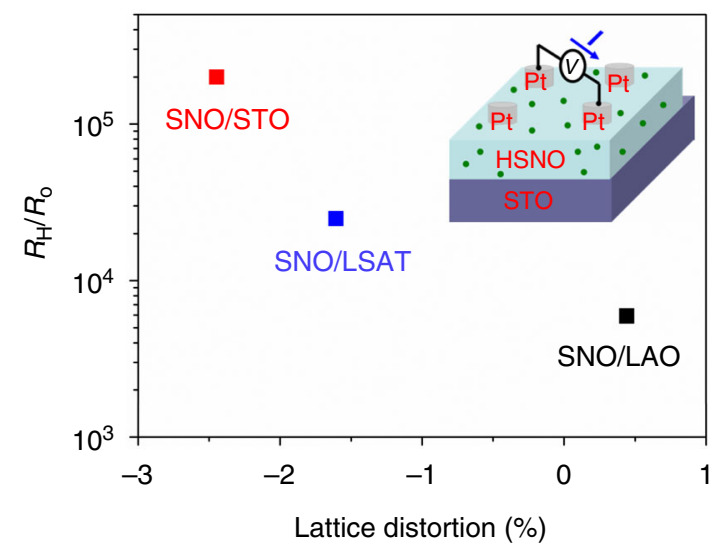

Fig. 3 Hydrogen profiles and resistivity of $\mathrm{H}_{x} \mathrm{SmNiO}_{3}$ upon distortions. a, $\mathbf{b}$ Hydrogen depth profiles for $\mathbf{a} \mathrm{SmNiO}_{3} / \mathrm{SrTiO}_{3}$ and $\mathbf{b ~ S m N i O} / \mathrm{LaAlO}$ in the $1 \%$ $\mathrm{H}_{2} / \mathrm{He}$ atmosphere after designed annealing durations. Black square: pristine $\mathrm{SmNiO}_{3}$; red dot: upon hydrogen annealing for 15 min; blue triangle: upon hydrogen annealing for $60 \mathrm{~min} \mathbf{c}$ Hydrogen concentration after annealing for $15 \mathrm{~min}$ and $\mathbf{d}$ the respective enhancements of the resistivity, plotted as a function of the lattice distortions for $\mathrm{SmNiO}_{3}$ grown on different single crystal substrates of $\mathrm{SrTiO}_{3}(\mathrm{red}),(\mathrm{La}, \mathrm{Sr})(\mathrm{Al}, \mathrm{Ta}) \mathrm{O}_{3}(\mathrm{blue})$, and $\mathrm{LaAlO}{ }_{3}$ (black)

$\mathrm{SmNiO}_{3}$ in oxygen (previously known as the dehydrogenation process), the more oxidizing atmosphere draws the chemical potential towards the formation of crystallized $\mathrm{SmNiO}_{3}{ }^{28}$, which may render the metastable state converting back to the ground state. Such a process is in a great analogy to crystallization of amorphous $\mathrm{SmNiO}_{3}$ when annealed at high oxygen pressures, as reported by refs. ${ }^{27-29}$ This understanding is in consistency to the previous observations that the potential oxygen vacancies generated in oxides when annealing in vacuum can also result in huge resistance change in oxide thin films, in which case the hydrogen composition is absent ${ }^{32,33}$. The tensile distortion further facilitates the formation of oxygen vacancies ${ }^{34,35}$, which may result in more significant enhancement in the resistivity of the $\mathrm{SmNiO}_{3}$ as also observed in this work.

For a more in-depth investigation of the enhanced resistivity via hydrogenation observed for tensile-strained $\mathrm{SmNiO}_{3}$, near edge Xray absorption fine structure (NEXAFS) $)^{36,37}$ analysis was performed for $\mathrm{SmNiO}_{3} / \mathrm{SrTiO}_{3}$ (001) and $\mathrm{SmNiO}_{3} / \mathrm{LaAlO}_{3}$. As reported previously, the orbital configuration associated to various valence states of $\mathrm{Ni}$ can be probed by comparing the NEXAFS spectrums of the Ni: $L$-edge and O: K-edge, as demonstrated in Fig. 4b. The Ni: $L$-edge usually contains two parts, while the Ni: $L_{3}$ originates from the $\mathrm{Ni} 2 \mathrm{p} \rightarrow \mathrm{Ni} 3 \mathrm{~d}$ transition, and usually splits into two peaks, reflecting the $t^{6}{ }_{2 \mathrm{~g}} e^{2} \mathrm{~g}\left(\mathrm{Ni}^{2+}\right.$, Peak $\left.\mathrm{A}\right)$ and $t^{6}{ }_{2 \mathrm{~g}} e^{1}{ }_{\mathrm{g}}\left(\mathrm{Ni}^{3+}\right.$,
Peak B), respectively ${ }^{36,38,39}$. Before hydrogenation, the tensiledistorted $\mathrm{SmNiO}_{3} / \mathrm{SrTiO}_{3}$ shows a reduced proportion in Peak B as compared to $\mathrm{SmNiO}_{3} / \mathrm{LaAlO}_{3}$, indicating a reduced proportion in the $t^{6}{ }_{2 \mathrm{~g}} \mathrm{e}_{\mathrm{g}}^{1}$ orbital configurations. The further hydrogenation treatment further reduces the proportion in Peak B in Ni: $L_{3}$ spectrums more significantly for $\mathrm{SmNiO}_{3} / \mathrm{SrTiO}_{3}$ compared to $\mathrm{SmNiO}_{3} / \mathrm{LaAlO}_{3}$, as shown in Supplementary Figure 10. These observations are further evidenced by a more significant reduction in the valance state of nickel for $\mathrm{SmNiO}_{3} / \mathrm{SrTiO}_{3}(001)$ as compared to $\mathrm{SmNiO}_{3} / \mathrm{LaAlO}_{3}$ demonstrated by the $\mathrm{X}$-ray photoelectron spectroscopy as shown in Supplementary Figure 11.

The O: $K$ spectrum exhibits a pre-peak A $(\sim 529 \mathrm{eV})$ that was attributed to the $\mathrm{Ni}: 3 d-\mathrm{O}: 2 p$ hybridization ( $d^{8} L$ configuration), and was previously used to monitor the oxygen vacancy formation 36,39 . The peaks appearing at the higher photon energies were expected to reflect the more oxygen depleted orbital configurations, i.e., $d^{9} L(\text { Peak } B)^{39}$. Before hydrogenation, the tensile-strained $\mathrm{SmNiO}_{3} / \mathrm{SrTiO}_{3}$ shows a smaller proportion of pre-peak A in O: $K$-edge, as compared to $\mathrm{SmNiO}_{3} / \mathrm{LaAlO}_{3}$. This is in agreement with the previous understanding that tensile distortion can result in weakened $\mathrm{Ni}-\mathrm{O}$ interactions when forming the bonding orbital, by either generating oxygen vacancies or reducing the valence state of $\mathrm{Ni}$. As a result, the construction of the tensile-distorted $\mathrm{NiO}_{6}$ octahedron is expected to be more 
a
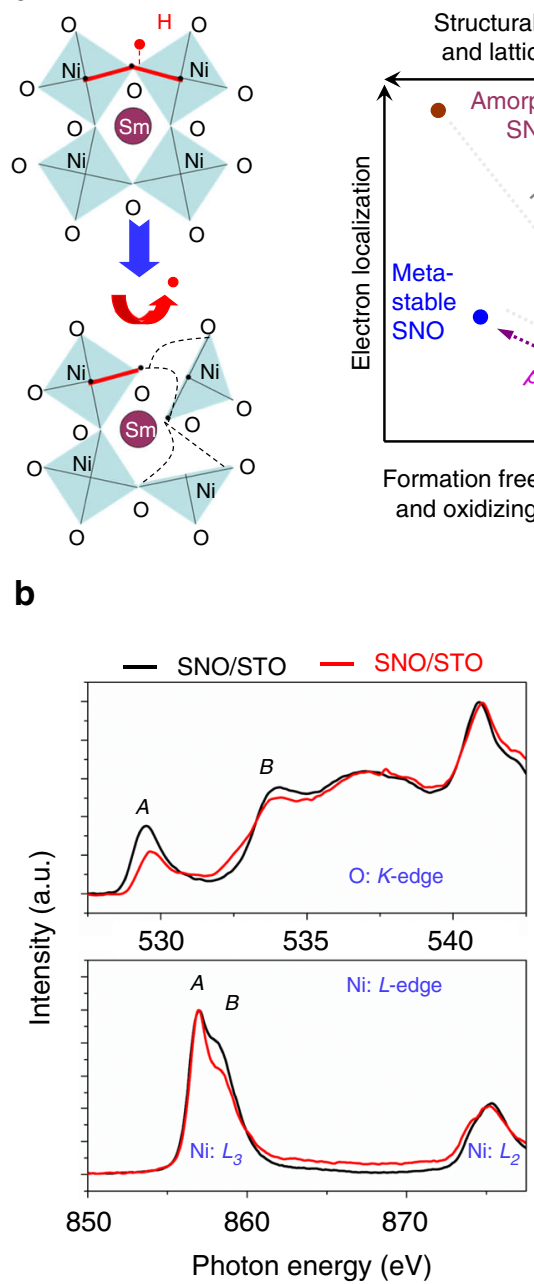

b

C

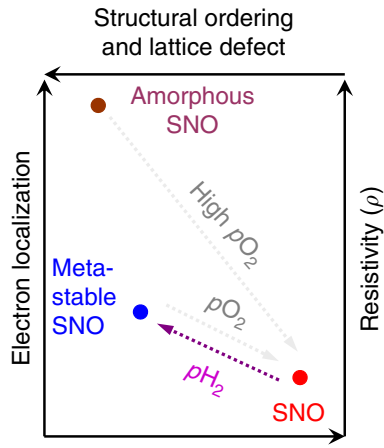

Formation free energy $(\Delta G)$ and oxidizing atmosphere

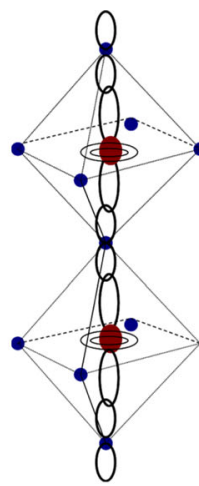

Conductive SNO

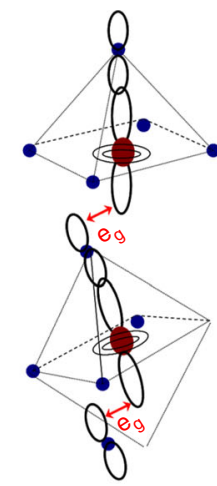

Insulating

H-SNO
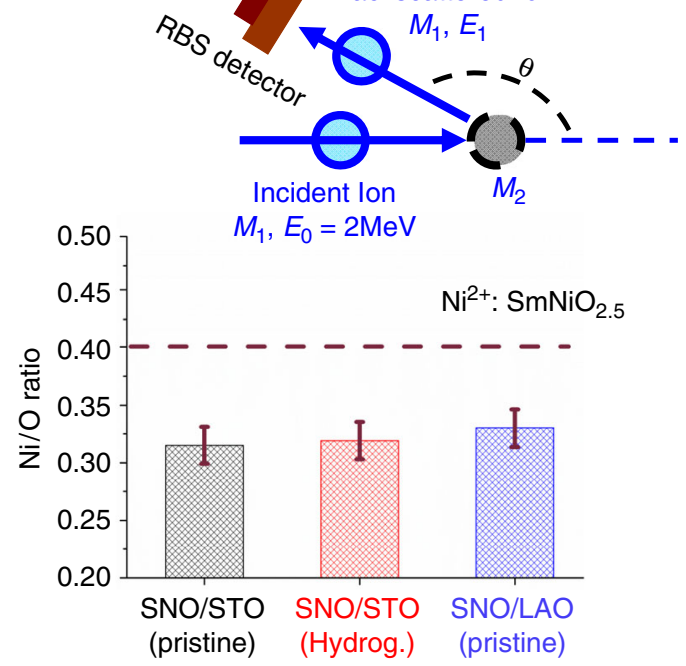

Fig. 4 Transient state in proton regulation of $\mathrm{SmNiO}_{3}$. a Illustrating the transition from the electron itinerant state to highly electron localized state triggered by the transient process during hydrogen diffusion, instead of the physical presence of the hydrogen composition. The structural disordering owing to lattice distortions results in the opening in band gap. b Near edge X-ray absorption fine structure (NEXAFS) analysis for $\mathrm{SmNiO}_{3} / \mathrm{SrTiO}_{3}($ red line) and $\mathrm{SmNiO}_{3} / \mathrm{LaAlO}_{3}$ (black line). c Ratios of the $\mathrm{Ni} / \mathrm{O}$ composition measured for $\mathrm{SmNiO} / \mathrm{SrTiO}_{3}$, hydrogenated $\mathrm{SmNiO} \mathrm{S}_{3} / \mathrm{SrTiO}_{3}$, and SmNiO $3 / \mathrm{LaAlO}$ by using the Rutherford backscattering (RBS). The upper scheme illustrates the RBS detection

easily destroyed by the hydrogen diffusion. Upon hydrogenation, a more significant reduction in the proportion of pre-peak $\mathrm{A}$ is observed in $\mathrm{O}: \mathrm{K}$-edge for $\mathrm{H}-\mathrm{SmNiO}_{3} / \mathrm{SrTiO}_{3}$ compared to $\mathrm{H}-\mathrm{SmNiO}_{3} / \mathrm{LaAlO}_{3}$ (see Supplementary Figure 10a and b).

The above observations are in agreement with our understanding that it is easier to cause deviations of the oxygen lattice atoms in the more tensile-distorted $\mathrm{SmNiO}_{3}$, resulting in more significant enhancement of the material resistivity. Nevertheless, the deviations of the oxygen lattice atoms do not necessarily deplete the oxygen composition and form conventional oxygen vacancies, in which case a composition of $\mathrm{SmNiO}_{2.5}$ is expected when $\mathrm{Ni}^{3+}$ is fully reduced to $\mathrm{Ni}^{2+}$. When measuring the thin film composition before and after hydrogenation via Rutherford backscattering (RBS), we observed no significant variations in the oxygen composition, as compared in Fig. 4c (see the RBS spectrum in Supplementary Figure 12). Therefore, the hydrogenation-induced electron localization in $\mathrm{SmNiO}_{3}$ is at least not simply associated to the depletion in oxygen composition to reduce nickel. In contrast, the kinetic process of hydrogenation (Supplementary Figure 13, Supplementary Table 1 and Supplementary Figure 14) is expected to form a thermodynamically more stabilized insulating state of $\mathrm{SmNiO}_{3}$ by driving the potential to reduce the pristine-positive formation free energy and without varying the chemical composition.

\section{Discussion}

The present understanding also supports the previous observations associated with hydrogenated $\mathrm{SmNiO}_{3}{ }^{1,2,5}$ from the kinetic perspective. For example, Zuo et al demonstrated that the formation of the insulating phase of $\mathrm{SmNiO}_{3}$ was related to the dynamic modulation and path during the hydrogenation, which is known to be a habituation-based plasticity behavior ${ }^{2}$. Such kinetic dependent behavior in the hydrogen-induced phase transition may be more associated with the variety in forming the metastable chemical state when altering the hydrogenation potential, rather than varying the hydrogen doping content. Similar kinetic dependencies were also recently observed by Shi et $\mathrm{al}^{1}$ and $\mathrm{Zhang}$ et $\mathrm{al}^{5}$, when performing the hydrogenation of $\mathrm{SmNiO}_{3}$ via hydrogen annealing or electrochemical approaches, respectively. In both situations, the resistivity of the hydrogeninduced insulating phase was elevated by improving the dynamic strength, i.e., elevating the temperature or enhancing the applied 
external voltage. Nevertheless, the specific mechanisms associated to the enhancement in resistivities of hydrogenated $\mathrm{VO}_{2}$ or $\mathrm{SrCoO}_{2.5}$ may be different to the one for $\mathrm{SmNiO}_{3}$, owing to the complexity of metal-insulator transitions for various correlated oxides.

In summary, we demonstrate quantitative and direct hydrogen detection within hydrogenated single crystalline $\mathrm{SmNiO}_{3}$ thin films-based nuclear resonance reactions between high-energy ${ }^{15} \mathrm{~N}^{2+}$ and ${ }^{1} \mathrm{H}$. By regulating the lattice mismatch between the film and substrate to impart tensile distortion up to $2.4 \%$ upon $\mathrm{SmNiO}_{3}$, the incorporated hydrogen concentration is reduced from $\sim 10^{21}$ to $\sim 10^{20} \mathrm{~cm}^{-3}$. Despite the reduction of the hydrogen concentration, a more significant hydrogen-induced enhancement in resistivity was observed for the more tensile-strained $\mathrm{SmNiO}_{3}$. Further combined results from near edge X-ray absorption fine structure and Rutherford backscattering analysis indicate that a more significant transition in the orbital configuration from an electron itinerant state to an electron-localized one is achieved in tensile-distorted $\mathrm{SmNiO}_{3}$ without dependence on the hydrogen concentration. Assisted by density functional theory calculations, this transition is demonstrated to be associated with a transient chemical state occurring in the transient diffusion process of hydrogen, despite the absence of hydrogen at the post-transition stage.

\section{Methods}

Sample preparation. Thin $\mathrm{SmNiO}_{3}$ thin films were grown on $\mathrm{SrTiO}_{3}(001)$, $\mathrm{LaAlO}_{3}(001)$, and $(\mathrm{La}, \mathrm{Sr})(\mathrm{Al}, \mathrm{Ta}) \mathrm{O}_{3}(001)$ single crystal substrates by pulsed laser deposition (PLD) at $20 \mathrm{~Pa} p \mathrm{O}_{2}$ and a substrate temperature of $650{ }^{\circ} \mathrm{C}$. For the hydrogenation treatment, the platinum electrodes were patterned on the surface of $\mathrm{SmNiO}_{3}$, followed by annealing in $1 \% \mathrm{H}_{2} / \mathrm{He}$ gas at $300{ }^{\circ} \mathrm{C}$ for $15-60 \mathrm{~min}$. More detailed descriptions associated for the sample preparation are presented in the Supplementary Methods.

Sample characterization. High-angle annular dark-field (HAADF) and annular bright-field (ABF) scanning transmission electron microscopy (STEM) experimental techniques were carried out on JEM-ARM $200 \mathrm{~F}$. The crystal structures were characterized by X-ray diffraction (XRD) and reciprocal space mapping (RSM). The diffraction patterns of [114] reciprocal space vectors from the film and substrate were projected at [110] and [001], representing the in-plane and crossplane reciprocal space vector, respectively. The resistance of as-grown thin films was measured in vacuum by using a commercialized CTA-system within the temperature range from $300-550 \mathrm{~K}$. The oxygen composition of the thin film was measured by Rutherford backscattering (RBS) in ETH Zurich by using a $2 \mathrm{MeV}$ ${ }^{4} \mathrm{He}$ beam and a silicon PIN diode detector at $\theta=168^{\circ}$. The collected RBS data were simulated using the RUMP software, and the uncertainty for the as-measured composition is within $5 \%$. The nuclear reaction analysis (NRA) measurement was performed in the Micro Analysis Laboratory, Tandem accelerator (MALT) at The University of Tokyo. More detailed descriptions with the sample characterization are given in the Supplementary Methods.

\section{Data availability}

The data that support the findings of this study are available from the corresponding authors upon reasonable request.

Received: 6 May 2018 Revised: 27 December 2018 Accepted: 22 January 2019

Published online: 11 February 2019

\section{References}

1. Shi, J., Zhou, Y. \& Ramanathan, S. Colossal resistance switching and band gap modulation in a perovskite nickelate by electron doping. Nat. Commun. 5, 4860 (2014).

2. Zuo, F. et al. Habituation based synaptic plasticity and organismic learning in a quantum perovskite. Nat. Commun. 8, 240 (2017).

3. Shi, J., Ha, S. D., Zhou, Y., Schoofs, F. \& Ramanathan, S. A correlated nickelate synaptic transistor. Nat. Commun. 4, 2676 (2013).

4. Zhou, Y. et al. Strongly correlated perovskite fuel cells. Nature 534, 231-234 (2016).
5. Zhang, Z. et al. Rerovskite nickelates as electric-field sensor in salt water. Nature 553, 68-72 (2018).

6. Lu, N. et al. Electric-field control of tri-state phase transformation with a selective dual-ion switch. Nature 546, 124-128 (2017).

7. Yoon, H. et al. Reversible phase modulation and hydrogen storage in multivalent $\mathrm{VO}_{2}$ epitaxial thin films. Nat. Mater. 15, 1113-1119 (2016).

8. Lozada-Hidalǵo, M. et al. Sieving hydrogen isotopes through two-dimensional crystals. Science 351, 68-70 (2016).

9. Paris, A. et al. Kinetic isotope effect in the hydrogenation and deuteration of graphene. Adv. Funct. Mater. 23, 1628-1635 (2013).

10. Myers, S. M. et al. Hydrogen interactions with defects in crystalline solids. Rev. Mod. Phys. 64, 559-617 (1992).

11. Song, J. \& Curtin, W. A. Atomic mechanism and prediction of hydrogen embrittlement in iron. Nat. Mater. 12, 145-151 (2012).

12. Xie, D. G. et al. In situ study of the initiation of hydrogen bubbles at the aluminium metal/oxide interface. Nat. Mater. 14, 899-903 (2015).

13. Li, M. et al. Effect of hydrogen on the integrity of aluminium-oxide interface at elevated temperatures. Nat. Commun. 8, 14564 (2016).

14. Gemma, R., Dobron, P., Cizek, J. \& Pundt, A. Stress release and defect occurrence in V1-xFex films upon hydrogen loading: H-induced superabundant vacancies, movement and creation of dislocations. Acta Mater. 67, 308-323 (2014).

15. Pálsson, G. K., Bliersbach, A., Wolff, M., Zamani, A. \& Hjörvarsson, B. Using light transmission to watch hydrogen diffuse. Nat. Commun. 3, 892 (2012).

16. Wilden, M. \& Fukutanin, K. Hydrogen detection near surfaces and shallow interfaces with resonant nuclear reaction analysis. Surf. Sci. Rep. 69, 196-295 (2014).

17. Mao, W. et al. Fabrication and hydrogen permeation properties of epitaxial $\mathrm{Er}_{2} \mathrm{O}_{3}$ films revealed by nuclear reaction analysis. J. Phys. Chem. C 120, 15147-15152 (2016).

18. Lanford, W. A., Trautvetter, H. P., Ziegler, J. F. \& Keller, J. New precision technique for measuring the concentration versus depth of hydrogen in solids. Appl. Phys. Lett. 28, 566 (1976).

19. Fluri, A. et al. Enhanced proton conductivity in $\mathrm{Y}$-Doped $\mathrm{BaZrO}_{3}$ via strain engineering. Adv. Sci. 4, 1700467 (2017).

20. Korte, C. et al. Coherency strain and its effect on ionic conductivity and diffusion in solid electrolytes - an improved model for nanocrystalline thin films and a review of experimental data. Phys. Chem. Chem. Phys. 16, 24575-24591 (2014).

21. Chen, Q. et al. Hydrostatic pressure decreases the proton mobility in the hydrated proton conductor. Appl. Phys. Lett. 97, 041902 (2010).

22. Bruno, F. Y. et al. Rationalizing strain engineering effects in rare-earth nickelates. Phys. Rev. B 88, 195108 (2013).

23. Shukla, N. et al. Electrically induced insulator to metal transition in epitaxial $\mathrm{SmNiO}_{3}$ thin films. Appl. Phys. Lett. 105, 012108 (2014).

24. Catalan, G. Progress in perovskite nickelate research. Phase Transit. 81, 729-749 (2008).

25. Conchon, F., Boulle, A. \& Guinebretière, R. Effect of tensile and compressive strains on the transport properties of $\mathrm{SmNiO}_{3}$ layers epitaxially grown on (001) $\mathrm{SrTiO}_{3}$ and $\mathrm{LaAlO}_{3}$ substrates. Appl. Phys. Lett. 91, 192110 (2007).

26. Scherwitzl, R. et al. Electric-Field Control of the Metal-insulator transition in ultrathin $\mathrm{NdNiO}_{3}$ films. Adv. Mater. 22, 5517-5520 (2010).

27. Chen, J. et al. Self-limited kinetics of electron doping in correlated oxides. Appl. Phys. Lett. 107, 031905 (2015)

28. Jaramillo, R., Schoofs, F., Ha, S. D. \& Ramanathan, S. High pressure synthesis of $\mathrm{SmNiO} 3$ thin films and implications for thermodynamics of the nickelates. J. Mater. Chem. C 1, 2455-2462 (2013).

29. Jaramillo, R., Ha, S. D., Silevitch, D. M. \& Ramanathan, S. Origins of badmetal conductivity and the insulator-metal transition in the rare-earth nickelates. Nat. Phys. 10, 304-307 (2014).

30. Chen, J. et al. Ultrahigh thermoelectric performance in $\mathrm{SrNb}_{0.2} \mathrm{Ti}_{0.8} \mathrm{O}_{3}$ oxide films at a submicrometer-scale thickness. ACS Energy Lett. 2, 915-921 (2017).

31. Conchon, F. et al. Investigation of strain relaxation mechanisms and transport properties in epitaxial films. J. Appl. Phys. 103, 123501 (2008).

32. Chandrasena, R. U. et al. Strain-engineered oxygen vacancies in $\mathrm{CaMnO}_{3}$ thin films. Nano. Lett. 17, 794-799 (2017).

33. Petrie, J. R. et al. Strain control of oxygen vacancies in epitaxial strontium cobaltite films. Adv. Funct. Mater. 26, 1564-1570 (2016).

34. Wang, L. et al. Oxygen Vacancy Induced room-temperature metal-insulator transition in nickelate films and its potential application in photovoltaics. ACS Appl. Mater. Interfaces 8, 9769-9776 (2016).

35. Xie, Y. et al. Control of functional responses via reversible oxygen loss in $\mathrm{La}_{1}$ ${ }_{\mathrm{x}} \mathrm{Sr}_{\mathrm{x}} \mathrm{FeO}_{3-\delta}$ films. Adv. Mater. 26, 1434-1438 (2014).

36. Kleiner, K. et al. Unraveling the Degradation Process of LiNi0.8Co0.15Al0.05O2 Electrodes in commercial lithium ion batteries by electronic structure investigations. ACS Appl. Mater. Interfaces 7, 19589-19600 (2015). 
37. Mossanek, R. J. O. et al. Effects of Ni vacancies and crystallite size on the $\mathrm{O}$ 1s and Ni 2p x-ray absorption spectra of nanocrystalline NiO. J. Phys. Condens. Matter 25, 495506 (2013).

38. Montoro, L. A., Abbate, M. \& Rosolen, J. M. Electronic structure of transition metal Ions in deintercalated and reintercalated $\mathrm{LiCo}_{0.5} \mathrm{Ni}_{0.5} \mathrm{O}_{2}$. J. Electrochem. Soc. 147, 1651-1657 (2000).

39. Kobayashi, H., Shikano, M., Koike, S., Sakaebe, H. \& Tatsumi, K. Investigation of positive electrodes after cycle testing of high-power Li-Ion battery cells. J. Power Sources 174, 380-386 (2007).

\section{Acknowledgements}

This work was supported by National Natural Science Foundation of China (No. 51602022 and No. 61674013). J.C. also appreciate Japanese Society for the Promotion of Science (Fellowship ID: P15363). Y.W. and J.S. were supported by the Rensselaer Polytechnic Institute Presidential Graduate Fellowship and the Air Force Office of Scientific Research under award number FA9550-18-1-0116. We appreciate helpful discussions and technical support from Prof. Akira Toriumi from The University of Tokyo (Japan), and Prof. Rafael Jaramillo from Massachusetts Institute of Technology (USA).

\section{Author contributions}

J.C., J.S., and Y.J. planned the present work; J.C. grew the thin films and performed the hydrogenation experiment; W.M. and H.M. performed the NRA experiment and analysed the data; B.G. performed the TEM experiment; J.W. managed the EXAFS experiment; V.W. and W.G. performed the DFT calculation; M.D. performed the RBS

measurement; J.C. and X.K. performed the RSM experiment and analysed the data. J.C., Y.W., and J.S. interpreted the transport and NRA results. J.C. wrote the manuscript. All authors discussed the results and commented on the manuscript.

\section{Additional information}

Supplementary Information accompanies this paper at https://doi.org/10.1038/s41467019-08613-3.

Competing interests: The authors declare no competing interests.

Reprints and permission information is available online at http://npg.nature.com/ reprintsandpermissions/

Journal peer review information: Nature Communications thanks Le Wang and the other anonymous reviewers for their contribution to the peer review of this work.

Publisher's note: Springer Nature remains neutral with regard to jurisdictional claims in published maps and institutional affiliations.

\section{(c) (i)}

Open Access This article is licensed under a Creative Commons Attribution 4.0 International License, which permits use, sharing, adaptation, distribution and reproduction in any medium or format, as long as you give appropriate credit to the original author(s) and the source, provide a link to the Creative Commons license, and indicate if changes were made. The images or other third party material in this article are included in the article's Creative Commons license, unless indicated otherwise in a credit line to the material. If material is not included in the article's Creative Commons license and your intended use is not permitted by statutory regulation or exceeds the permitted use, you will need to obtain permission directly from the copyright holder. To view a copy of this license, visit http://creativecommons.org/ licenses/by/4.0/.

(C) The Author(s) 2019 